\title{
GENERALIZED HO-KALMAN ALGORITHM FOR 2D CONTINUOUS DISCRETE LINEAR SYSTEMS
}

\author{
V. Prepeliţă \\ Universitatea Politehnica Bucureşti, Catedra Matematici I, \\ Splaiul Independentei 313, Sector 6, Cod 77206, Bucureşti, Romania \\ vprepelita@pcnet.ro
}

\begin{abstract}
A class of continuous-discrete time-variable linear control systems is considered, whose state space representation is a system of differential equations with respect to one variable and of difference equations with respect to the second one. The fundamental concepts of reachability and observability are analysed in this framework. In the case of timeinvariant systems the structure of the transfer matrix is obtained and some properties of minimal realizations are emphasized. The connection between reachability, observability and minimality is established. An algorithm is proposed which provides a minimal realization for multiinput-multi-output systems. This method generalizes to $2 \mathrm{D}$ systems the celebrated Ho-Kalman algorithm.
\end{abstract}

MSC 1991: 93B15, 93B20, 93C35

Keywords. 2D continuous-discrete systems, controllability, observability, minimal realizations.

\section{Introduction}

Last years proved an increasing interest in the study of multivariable systems, determined by their wide range of applications in domains like image processing, geophysics and seismology, computer tomography etc.

One of the most important problems in this approach is that of the minimal state-space realization of $n D$ transfer matrices. As it was emphasized in B. De Schutter's overview [1], the notion of minimality plays a powerful role in the analysis and design of $n D$ digital signal processing. It has been proved that minimal state space realizations are possible only in special cases of rational $2 D$ transfer functions with separable denominator, separable numerator, for the all-pole and all-zero systems or for the continued fraction expansions (see [2], [3] and [4]). The disadvantage

The original version of this chapter was revised: The copyright line was incorrect. This has been corrected. The Erratum to this chapter is available at DOI: 10.1007/978-0-387-35690-7_44

V. Barbu et al. (eds.), Analysis and Optimization of Differential Systems

(C) IFIP International Federation for Information Processing 2003 
of the existing algorithms is their restriction to single-input-single-output (SISO) systems.

In this paper an algorithm is proposed which determines a minimal realization for separable $2 D$ multi-input-multi-output (MIMO) systems. This method generalizes to $2 D$ systems the celebrated Ho-Kalman algorithm (see [5]) which gave rise to many interesting developments (see [1] and [6]). A similar procedure was proposed in [7] in Kalman's module theoretic approach.

The basic concepts of controllability and observability are discussed in the general case of time-variable systems and suitable Gramians and matrices are defined. The connection between the controllability matrix, the observability matrix and the Hankel matrix of a $2 D$ time-invariant system is emphasized and it is used to prove the minimality of the provided realization.

The above topics are related to a class of continuous-discrete linear control systems whose state space representation is a system of differential equations with respect to one variable and of difference equations with respect to the second one. This class corresponds to Attasi's two dimensional discrete-time linear systems (see [8]), and in the time-invariant case was studied in [9]. Similar hybrid systems (but of Roesser [10] and Fornasini-Marchesini [11] type) were studied in a series of papers due to Kaczorek (see [12], [13] and [14]). The study of $2 D$ continuous-discrete systems is motivated by their applications in various domains like discrete linear repetitive processes (or multipass processes), in iterative learning control or in the study of linear systems with delays (see [15], [16] and [17]).

The proposed algorithm can also be used for MIMO separable $2 D$ discrete-time linear systems or for MIMO $2 D$ systems described by a class of hyperbolic partial differential equations.

\section{Two-Dimensional Continuous-Discrete Linear Systems}

The linear spaces $X=\mathbf{R}^{n}, U=\mathbf{R}^{m}$ and $Y=\mathbf{R}^{p}$ are called respectively the state, input and output spaces and $T=\mathbf{R} \times \mathbf{Z}$ is the time set.

Definition 2.1. A two-dimensional continuous-discrete linear system (2Dcd) is a quintuplet $\Sigma=\left(A_{1}(t, k), A_{2}(t, k), B(t, k), C(t, k), D(t, k)\right) \in$ $\mathbf{R}^{n \times n_{\times}} \mathbf{R}^{n \times n_{\times}} \mathbf{R}^{n \times m_{\times}} \times \mathbf{R}^{p \times n_{\times}} \mathbf{R}^{p \times m}$ with $A_{1}(t, k) A_{2}(t, k)=A_{2}(t, k) A_{1}(t, k)$ $\forall(t, k) \in T$, where all matrices are continuous with respect to $t \in \mathbf{R}$ for any $k \in \mathbf{Z}$; the state space representation of $\Sigma$ is given by the state and 
output equations

$$
\begin{array}{r}
\dot{x}(t, k+1)=A_{1}(t, k+1) x(t, k+1)+A_{2}(t, k) \dot{x}(t, k)- \\
-A_{1}(t, k) A_{2}(t, k) x(t, k)+B(t, k) u(t, k) \\
y(t, k)=C(t, k) x(t, k)+D(t, k) u(t, k)
\end{array}
$$

where $\dot{x}(t, k)=\frac{\partial x}{\partial t}(t, k)$. The number $n$ is called the dimension of the system $\Sigma$ and it is denoted by $\operatorname{dim} \Sigma$.

All the following results remain valid in the more general context of matrices over spaces of functions of bounded variation or regulated functions with respect to $t$, in which case the Perron-Stieltjes integral is used (see [18], [19] and [20]).

The (continuous) fundamental matrix of $A_{1}(t, k)$ with respect to $t \in \mathbf{R}$ is denoted by $\Phi\left(t, t_{0} ; k\right)$ for any fixed $k \in \mathbf{Z}$; therefore $\Phi\left(t, t_{0} ; k\right)$ is the unique matrix solution of the system $\dot{Y}(t, k)=A_{1}(t, k) Y(t, k), Y\left(t_{0}, k\right)=I$. If $A_{1}$ is a constant matrix, then $\Phi\left(t, t_{0} ; k\right)=e^{A_{1}\left(t-t_{0}\right)}$.

The discrete fundamental matrix $F\left(t ; k, k_{0}\right)$ of the matrix $A_{2}(t, k)$ is defined for any fixed $t \in \mathbf{R}$ by

$$
F\left(t ; k, k_{0}\right)=\left\{\begin{array}{ccc}
A_{2}(t, k-1) A_{2}(t, k-2) \ldots A_{2}\left(t, k_{0}\right) & \text { for } & k>k_{0} \\
I_{n} & \text { for } & k=k_{0} .
\end{array}\right.
$$

$F\left(t ; k, k_{0}\right)$ is the unique matrix solution of the system of difference equations $Y(t, k+1)=A_{2}(t, k) Y(t, k), Y\left(t, k_{0}\right)=I$. If $A_{2}$ is a constant matrix, then $F\left(t ; k, k_{0}\right)=A_{2}^{k-k_{0}}$.

Since $A_{1}(t, k)$ and $A_{2}(t, k)$ are commutative matrices for any $(t, k) \in T$, it results by Peano-Baker formula for $\Phi$ and by the definition of $F$ that $\Phi\left(t, t_{0} ; k\right)$ and $F\left(s ; l, l_{0}\right)$ are commutative matrices for any $t, t_{0}, s \in \mathbf{R}$ and $k, l, l_{0} \in \mathbf{Z}$.

Definition 2.2. A vector $x_{0} \in X$ is said to be the initial state of $\Sigma$ at the moment $\left(t_{0}, k_{0}\right) \in T$ if for any $(t, k) \in T$ with $(t, k) \geq\left(t_{0}, k_{0}\right)$ the following conditions hold:

$$
x\left(t, k_{0}\right)=\Phi\left(t, t_{0} ; k_{0}\right) x_{0} \quad x\left(t_{0}, k\right)=F\left(t_{0} ; k, k_{0}\right) x_{0}
$$

For some $\left(t_{0}, k_{0}\right),(t, k) \in T$ with $\left(t_{0}, k_{0}\right)<(t, k)$ we denote by $I$ the set $I=\left[t_{0}, t\right] \times\left[k_{0}, k\right], I \subset T$. An input function or a control is a function $u: I \rightarrow U$ such that $u(\cdot, k)$ is continuous for any $k \in \mathbf{Z}$.

In [21] it was proved that the state of $\Sigma$ at the moment $(t, k) \in T$ determined by the initial state $x_{0}$ and by the input function $u$ is given by

$$
\begin{gathered}
x(t, k)=\Phi\left(t, t_{0} ; k\right) F\left(t_{0} ; k, k_{0}\right) x_{0}+ \\
+\int_{t_{0}}^{t} \sum_{l=k_{0}}^{k-1} \Phi(t, s ; k) F(s ; k, l+1) B(s, l) u(s, l) \mathrm{d} s .
\end{gathered}
$$


By replacing the state $x(t, k)$ given by (2.4) into the output equation (2.2) we obtain the input-output map of the system $\Sigma$ :

$$
\begin{gathered}
y(t, k)=C(t, k) \Phi\left(t, t_{0} ; k\right) F\left(t_{0} ; k, k_{0}\right) x_{0}+ \\
+\int_{t_{0}}^{t} \sum_{l=k_{0}}^{k-1} C(t, k) \Phi(t, s ; k) F(s ; k, l+1) B(s, l) u(s, l) \mathrm{d} s+D(t, k) u(t, k) .
\end{gathered}
$$

Definition 2.3. A triplet $(t, k, x) \in \mathbf{R} \times \mathbf{Z} \times X$ is said to be a phase of $\Sigma$ if $x$ is the state of $\Sigma$ at the moment $(t, k)$ (i.e. if $x=x(t, k)$, where $x(t, k)$ is given by $(2.4))$.

$$
\text { By }(s, l)<(t, k) \text { we mean } s \leq t, l \leq k \text { and }(s, l) \neq(t, k) \text {. }
$$

Definition 2.4. A phase $(t, k, x)$ of $\Sigma$ is said to be reachable if there exist $\left(t_{0}, k_{0}\right) \in T,\left(t_{0}, k_{0}\right)<(t, k)$ and a control $u(\cdot, \cdot)$ which transfers the phase $\left(t_{0}, k_{0}, 0\right)$ to $(t, k, x)$.

A phase $(t, k, x)$ is said to be controllable if there exist $\left(t_{1}, k_{1}\right) \in T$, $\left(t_{1}, k_{1}\right)>(t, k)$ and a control $u(\cdot, \cdot)$ which transfers the phase to $(t, k, x)$ to $\left(t_{1}, k_{1}, 0\right)$.

If for some fixed $\left(t_{0}, k_{0}\right) \in T,\left(t_{1}, k_{1}\right) \in T,\left(t_{0}, k_{0}\right)<\left(t_{1}, k_{1}\right)$, every phase $\left(t_{1}, k_{1}, x\right)\left(\left(t_{0}, k_{0}, x\right)\right)$ is reachable (controllable) during the period $\left[t_{0}, t_{1}\right] \times\left[k_{0}, k_{1}\right] \subset \mathbf{R} \times \mathbf{Z}$, the system $\Sigma$ is said to be completely reachable (completely controllable) on $\left[t_{0}, t_{1}\right] \times\left[k_{0}, k_{1}\right]$.

The symmetrical non-negative definite $n \times n$ matrix

$$
\begin{gathered}
R_{\Sigma}\left(t_{0}, t ; k_{0}, k\right)= \\
\int_{t_{0}}^{t} \sum_{l=k_{0}}^{k-1} \Phi(t, s ; k) F(s ; k, l+1) B(s, l) B(s, l)^{T} F(s ; k, l+1)^{T} \Phi(t, s ; k)^{T} \mathrm{~d} s
\end{gathered}
$$

is called the reachability Gramian of $\Sigma$.

In [21] it was proved:

Proposition 2.5. The set of all states which are reachable on $I$ is the linear space $\operatorname{Im} R_{\Sigma}\left(t_{0}, t ; k_{0}, k\right)$.

Then we get

Theorem 2.6. $\Sigma$ is completely reachable on I if and only if

$$
\operatorname{Rank} R_{\Sigma}\left(t_{0}, t ; k_{0}, k\right)=n .
$$

Proof. By Proposition 2.5, $\Sigma$ is completely reachable on $I$ iff $\operatorname{Im} R_{\Sigma}\left(t_{0}, t ; k_{0}, k\right)=\mathbf{R}^{n}$, condition equivalent to $(2.7)$. 
Definition 2.7. A phase $\left(t_{0}, k_{0}, x\right)$ is said to be unobservable (unobservable on $I$ ) if for any control $u: I \rightarrow \mathbf{R}^{m}$ it provides the same output $y(s, l)$ for $(s, l) \geq(t, k)$ (for $(s, l) \in I)$ as the phase $\left(t_{0}, k_{0}, 0\right)$.

The system $\Sigma$ is said to be completely observable (completely observable on $I$ ) if there is no unobservable (unobservable on $I$ ) state $x \neq 0$.

In order to check whether a system $\Sigma$ is completely observable we introduce the 2D observability Gramian of $\Sigma$ denoted by $\mathcal{O}_{\Sigma}\left(t, t_{0} ; k, k_{0}\right)$ :

$$
\begin{gathered}
\mathcal{O}_{\Sigma}\left(t, t_{0} ; k, k_{0}\right)= \\
=\int_{t_{0}}^{t} \sum_{l=k_{0}}^{k} F\left(t_{0} ; l, k_{0}\right)^{T} \Phi\left(s, t_{0} ; l\right)^{T} C(s, l)^{T} C(s, l) \Phi\left(s, t_{0} ; l\right) F\left(t_{0} ; l, k_{0}\right) \mathrm{d} s .
\end{gathered}
$$

The following results are proved in [22]:

Proposition 2.8. The set of states which are unobservable on $I$ is the subspace $\operatorname{Ker} \mathcal{O}_{\Sigma}\left(t, t_{0} ; k, k_{0}\right)$.

Theorem 2.9. The system $\Sigma=\left(A_{1}(t, k), A_{2}(t, k), B(t, k), C(t, k)\right.$, $D(t, k))$ is completely observable on $I$ if and only if

$$
\operatorname{rank} \mathcal{O}_{\Sigma}\left(t, t_{0} ; k, k_{0}\right)=n .
$$

\section{Time-Invariant 2Dcd Systems}

The system $\Sigma=\left(A_{1}, A_{2}, B, C, D\right)$ is said to be time invariant if $A_{1}, A_{2}, B, C$ and $D$ are constant matrices. In this case the state formula (2.4) and the output formula (2.5) become

$$
\begin{gathered}
x(t, k)=e^{A_{1}\left(t-t_{0}\right)} A_{2}^{k-k_{0}} x_{0}+\int_{t_{0}}^{t} \sum_{l=k_{0}}^{k-1} e^{A_{1}(t-s)} A_{2}^{k-l-1} B u(s, l) \mathrm{d} s \\
y(t, k)=C e^{A_{1} t} A_{2}^{k} x_{0}+\int_{0}^{t} \sum_{l=0}^{k-1} C e^{A_{1}(t-s)} A_{2}^{k-l-1} B u(s, l) \mathrm{d} s+D u(t, k) .
\end{gathered}
$$

Since $\Sigma$ is time-invariant, we can consider the initial moment $\left(t_{0}, k_{0}\right)=(0,0)$ and the time set $T=\mathbf{R}^{+} \times \mathbf{Z}^{+}$.

We associate to $\Sigma$ the controllability matrix

$$
\begin{aligned}
& C_{\Sigma}=\left[\begin{array}{llllllll}
B & A_{1} B & \ldots & A_{1}^{n-1} B & A_{2} B & A_{1} A_{2} B & \ldots & A_{1}^{n-1} A_{2} B \ldots
\end{array}\right. \\
& \left.A_{2}^{n-1} B \quad A_{1} A_{2}^{n-1} B \quad \ldots A_{1}^{n-1} A_{2}^{n-1} B\right] .
\end{aligned}
$$

From Theorem 2.6 we get (see [21])

Theorem 3.1. $\Sigma$ is completely reachable if and only if

$$
\operatorname{rank} C_{\Sigma}=n .
$$


Theorem 3.2. The set of all reachable states of $\Sigma$ is the smallest subspace of $X$ which is $\left(A_{1}, A_{2}\right)$-invariant and contains the columns of $B$.

The matrix

$$
\begin{aligned}
& O_{\Sigma}=\left[\begin{array}{llllll}
C^{T} & A_{1}^{T} C^{T} & \ldots & \left(A_{1}^{T}\right)^{n-1} C^{T} & A_{2}^{T} C^{T} & A_{1}^{T} A_{2}^{T} C^{T}
\end{array} \ldots\left(A_{1}^{T}\right)^{n-1} A_{2}^{T} C^{T} \ldots\right. \\
& \left.\left(A_{2}^{T}\right)^{n-1} C^{T} A_{1}^{T}\left(A_{2}^{T}\right)^{n-1} C^{T} \ldots\left(A_{1}^{T}\right)^{n-1}\left(A_{2}^{T}\right)^{n-1} C^{T}\right]^{T}
\end{aligned}
$$

is called the observability matrix of the system $\Sigma$.

In [22] it was proved:

Theorem 3.3. The system $\Sigma=\left(A_{1}, A_{2}, B, C, D\right)$ is completely observable if and only if

$$
\operatorname{rank} O_{\Sigma}=n
$$

The following statement results from Theorems 3.1 and 3.3 by noticing that the $2 \mathrm{D}$ observability matrix $O_{\Sigma}$ of the system $\Sigma=\left(A_{1}, A_{2}, B, C, D\right)$ coincides with the $2 \mathrm{D}$ controllability matrix $C_{\Sigma^{*}}$ of the system $\Sigma^{*}=\left(A_{1}^{T}, A_{2}^{T}, C^{T}, B^{T}, D^{T}\right)$. We say that the system $\Sigma^{*}$ is the dual of $\Sigma$.

Theorem 3.4. The system $\Sigma$ is completely observable if and only if its dual $\Sigma^{*}$ is completely reachable.

By duality we obtain (see [22]):

Theorem 3.5. The set $X_{u o}$ of all unobservable states of $\Sigma$ is the greatest subspace of $X$ which is $\left(A_{1}, A_{2}\right)$-invariant and is contained in $\operatorname{Ker} C$.

\section{Transfer Matrix of 2Dcd Systems}

Let us consider the time-invariant 2Dcd system $\Sigma=\left(A_{1}, A_{2}, B, C, D\right)$. The initial conditions (2.3) become (with $x_{0}=x(0,0)$ )

$$
x(t, 0)=e^{A_{1} t} x_{0}, \forall t \in \mathbf{R}^{+} ; x(0, k)=A_{2}^{k} x_{0}, \forall k \in \mathbf{Z}^{+},
$$

hence

$$
\dot{x}(t, 0)=A_{1} x(t, 0) ; x(0, k+1)=A_{2} x(0, k) .
$$

We denote by $\tilde{x}(s, k)$ the Laplace Transform of the function $x(t, k)$ for $k \in \mathbf{Z}^{+}$and by $X(s, z)$ the $z$-Transform of $\tilde{x}(s, k)$ for $s \in \mathbf{C}$. The Differentiation-of-the-original Theorem for the Laplace Transformation and Second-delay Theorem for z-Transformation give

$s \tilde{x}(s, 0)-x(0,0)=A_{1} \tilde{x}(s, 0) ; z X(0, z)-z x(0,0)=A_{2} X(0, z)$.

By applying the Laplace Transformation to (2.1) we get $s \tilde{x}(t, k+1)-$ $x(0, k+1)=A_{1} \tilde{x}(s, k+1)+A_{2}(s \tilde{x}(s, k)-x(0, k))-A_{1} A_{2} \tilde{x}(s, k)+B \tilde{u}(s, k)$, 
equation in which we can reduce $x(0, k+1)$ by (4.2). Then we take the zTransform of the reduced equation and we obtain $z(s X(s, z)-s \tilde{x}(s, 0)=$ $A_{1} z(X(s, z)-\tilde{x}(s, 0))+A_{2} s X(s, z)-A_{1} A_{2} X(s, z)+B U(s, z)$ which gives by (4.3) $\left(z s I-A_{1}-A_{2}+A_{1} A_{2}\right) X(s, z)=B U(s, z)+z x_{0}$.

For $s \in \mathbf{C} \backslash \sigma\left(A_{1}\right), z \in \mathbf{C} \backslash \sigma\left(A_{2}\right)$ we have

$X(s, z)=\left(s I-A_{1}\right)^{-1}\left(z I-A_{2}\right)^{-1} B U(s, z)+\left(s I-A_{1}\right)^{-1}\left(z I-A_{2}\right)^{-1} z x_{0}$.

Both Laplace and $\mathrm{z}$ Transforms applied to $(2.2)$ give $Y(s, z)=C X(s, z)+$ $D U(s, z)$ and by replacing $X(s, z)(4.4)$ we obtain the input-output map of $\Sigma$ in the frequency domain

$$
\begin{gathered}
Y(s, z)= \\
{\left[C\left(s I-A_{1}\right)^{-1}\left(z I-A_{2}\right)^{-1} B+D\right] U(s, z)+C\left(s I-A_{1}\right)^{-1}\left(z I-A_{2}\right)^{-1} z x_{0} .}
\end{gathered}
$$

Definition 4.1. The matrix

$$
T_{\Sigma}(s, z)=C\left(s I-A_{1}\right)^{-1}\left(z I-A_{2}\right)^{-1} B+D
$$

is called the transfer matrix of $\Sigma$.

Obviously, $T_{\Sigma}(s, z)$ is a $p \times m$ rational proper (in both variables $s$ and $z$ ) matrix with separable denominator, since it has the form

$$
T_{\Sigma}(s, z)=\frac{1}{\operatorname{det}\left(s I-A_{1}\right) \operatorname{det}\left(s I-A_{2}\right)} C\left(s I-A_{1}\right)^{*}\left(z I-A_{2}\right)^{*} B+D .
$$

With $x_{0}=0$ in (4.5) we get

Proposition 4.2. The null state response of the system $T_{\Sigma}(s, z)$ in the frequency domain has the form $Y(s, z)=T_{\Sigma}(s, z) U(s, z)$ where $T_{\Sigma}(s, z)$ is the transfer matrix of $\Sigma$.

Let us denote by $P S(m, p)$ the set of $p \times m$ proper matrices with separable denominator and by $S P S(m, p)$ the set of strictly proper matrices in $P S(m, p)$.

Definition 4.3. Given $T(s, z) \in P S(m, p)$, a system $\Sigma=\left(A_{1}, A_{2}, B, C, D\right)$ is said to be a realization of $\Sigma$ if $T(s, z)=T_{\Sigma}(s, z)$, that is if

$$
T(s, z)=C\left(s I-A_{1}\right)^{-1}\left(z I-A_{2}\right)^{-1} B+D .
$$

A realization $\Sigma$ of $T(s, z)$ is minimal if $\operatorname{dim} \Sigma \leq \operatorname{dim} \tilde{\Sigma}$ for any realization $\tilde{\Sigma}$ of $T(s, z)$.

Since by (4.7) $D=\lim _{s \rightarrow \infty} T(s, z)=\lim _{z \rightarrow \infty} T(s, z)$, we can state the usual realization problem: given a strictly proper matrix $T(s, z)$ determine the quadruplet $\Sigma=\left(A_{1}, A_{2}, B, C\right)$ such that

$$
T(s, z)=C\left(s I-A_{1}\right)^{-1}\left(z I-A_{2}\right)^{-1} B .
$$


Now let us consider the Laurent series expansion of $T(s, z) \in S P S(p, m)$ about $s=\infty, z=\infty$

$$
T(s, z)=\sum_{i=0}^{\infty} \sum_{j=0}^{\infty} M_{i, j} s^{-i-1} z^{-j-1} .
$$

The matrices $M_{i, j} \in \mathbf{R}^{p \times m}$ are called the Markov parameters of $T(s, z)$.

Proposition 4.4. $\Sigma=\left(A_{1}, A_{2}, B, C\right)$ is a realization of $T(s, z)$ if and only if, for any $i, j \in \mathbf{N}$,

$$
M_{i, j}=C A_{1}^{i} A_{2}^{j} B .
$$

Proof. By (4.7) we have $T(s, z)=C\left(s I-A_{1}\right)^{-1}\left(z I-A_{2}\right)^{-1} B=$ $C\left(\sum_{i=0}^{\infty} A_{1}^{i} s^{-i-1}\right)\left(\sum_{j=0}^{\infty} A_{2}^{j} z^{-j-1}\right) B=\sum_{i=0}^{\infty} \sum_{j=0}^{\infty} C A_{1}^{i} A_{2}^{j} s^{-i-1} z^{-j-1}$. Since (4.9) holds and two equal Laurent series have equal corresponding coefficients, (4.10) is true.

The following theorem establishes the connection between the concepts of reachability, observability and minimality (the proof is omitted because of lack of space).

Theorem 4.5. A system $\Sigma=\left(A_{1}, A_{2}, B, C\right)$ is a minimal realization of some $T(s, z) \in S P S(p, m)$ if and only if $\Sigma$ is completely reachable and completely observable.

\section{Minimal realizations}

Let us consider a matrix $T(s, z) \in S P S(p, m)$ and its Markov parameters $M_{i, j}, i, j \geq 0$ given by (4.9). We associate to $T(s, z)$ the block Hankel matrices

$$
H_{k}^{j}=\left[\begin{array}{cccc}
M_{0, j} & M_{1, j} & \ldots & M_{k-1, j} \\
M_{1, j} & M_{2, j} & \ldots & M_{k, j} \\
\cdot & \cdot & \ldots & \cdot \\
M_{k-1, j} & M_{k, j} & \ldots & M_{2 k-2, j}
\end{array}\right], H_{k, l}=\left[\begin{array}{cccc}
H_{k}^{0} & H_{k}^{1} & \ldots & H_{k}^{l-1} \\
H_{k}^{1} & H_{k}^{2} & \ldots & H_{k}^{l} \\
\cdot & \cdot & \ldots & \cdot \\
H_{k}^{l-1} & H_{k}^{l} & \ldots & H_{k}^{2 l-2}
\end{array}\right] .
$$

Proposition 5.1. For any realization $\Sigma$ of $T(s, z)$ and any $k, l \geq 1$, $\operatorname{rank} H_{k, l} \leq \operatorname{dim} \Sigma$.

Proof. For some realization $\Sigma$ we denote by $C_{k, l}$ and $O_{k, l}$ the matrices with similar structures as $C_{\Sigma}$ (3.3) and $O_{\Sigma}$ (3.5) but with $k l$ blocks. Then $O_{k, l} C_{k, l}=H_{k, l}$ and by the second Sylvester Inequality we have for any $k, l \geq 1, \operatorname{rank} H_{k, l} \leq \min \left(\operatorname{rank} O_{k, l}, \operatorname{rank} C_{k, l}\right) \leq \operatorname{dim} \Sigma$. 
Now let $p_{1}(s) p_{2}(z)$ be the separable least common denominator of entries of $T(s, z)$, where $p_{1}(s)=s^{q}+\sum_{i=0}^{q-1} \alpha_{i} s^{i}, p_{2}(z)=z^{r}+\sum_{j=0}^{r-1} \beta_{j} z^{j}$. Let us consider the shift operators $\widetilde{\sigma}_{1}$ (first level), $\sigma_{1}$ and $\sigma_{2}$ (second level) applied to the two level block Hankel matrix $H_{q, r}$, defined by

$$
\begin{aligned}
\widetilde{\sigma}_{1} H_{q}^{j}= & {\left[\begin{array}{cccc}
M_{1, j} & M_{2, j} & \ldots & M_{q, j} \\
M_{2, j} & M_{3, j} & \ldots & M_{q+1, j} \\
\cdot & \cdot & \ldots & \cdot \\
M_{q, j} & M_{q+1, j} & \ldots & M_{2 q-1, j}
\end{array}\right] } \\
\sigma_{1} H_{q, r}= & {\left[\begin{array}{cccc}
\widetilde{\sigma}_{1} H_{q}^{0} & \widetilde{\sigma}_{1} H_{q}^{1} & \ldots & \widetilde{\sigma}_{1} H_{q}^{r-1} \\
\widetilde{\sigma}_{1} H_{q}^{1} & \widetilde{\sigma}_{1} H_{q}^{2} & \ldots & \widetilde{\sigma}_{1} H_{q}^{r} \\
\cdot & \cdot & \ldots & \cdot \\
\widetilde{\sigma}_{1} H_{q}^{r-1} & \widetilde{\sigma}_{1} H_{q}^{r} & \ldots & \widetilde{\sigma}_{1} H_{q}^{2 r-2}
\end{array}\right] } \\
\sigma_{2} H_{q, r}= & {\left[\begin{array}{cccc}
H_{q}^{1} & H_{q}^{2} & \ldots & H_{q}^{r} \\
H_{q}^{2} & H_{q}^{3} & \ldots & H_{q}^{r+1} \\
\cdot & \cdot & \ldots & \cdot \\
H_{q}^{r} & H_{q}^{r+1} & \ldots & H_{q}^{2 r-1}
\end{array}\right] . }
\end{aligned}
$$

Let $J_{k}$ be the companion cell associated to the polynomial $p_{k}, k=1,2$; we introduce the matrices

$$
\begin{array}{ll}
F_{1}=I_{r} \otimes J_{1} \otimes I_{p}, & F_{2}=J_{2} \otimes I_{q} \otimes I_{p} \\
\widetilde{F}_{1}=I_{r} \otimes J_{1}^{T} \otimes I_{m}, & \widetilde{F}_{2}=J_{2}^{T} \otimes I_{q} \otimes I_{m}
\end{array}
$$

where $\otimes$ denotes the Kronecker product.

Since $F_{1} F_{2}=\left(I_{r} J_{2}\right) \otimes\left(J_{1} I_{q}\right) \otimes\left(I_{p} I_{p}\right)=F_{2} F_{1}, F_{1}, F_{2}$ and similarly $\widetilde{F}_{1}, \widetilde{F}_{2}$ are commutative matrices.

\section{Proposition 5.2.}

$$
\sigma_{k} H_{q, r}=F_{k} H_{k, r}=H_{q, r} \widetilde{F}_{k}, k=1,2 .
$$

Proof. Since $p_{1}(s) T(s, z)$ and $p_{2}(z) T(s, z)$ are polynomial matrices with respect to $s$ and $z$ respectively, if we replace $T(s, z)$ by its Laurent series (4.9) and we equalize with zero the coefficients of the negative powers of $s$ and $z$ we get two recurrence relations:

$$
M_{l+q, j}=-\sum_{i=0}^{q-1} \alpha_{i} M_{l+i, j}, \forall l, j \geq 0 ; M_{i, l+r}=-\sum_{j=0}^{r-1} \beta_{j} M_{i, l+j}, \forall l, i \geq 0 .
$$

Equalities (5.4) result by a long but direct calculus, based on these relations.

By induction we get 


\section{Corollary 5.3.}

$$
\sigma_{k}^{h} H_{q, r}=F_{k}^{h} H_{k, r}=H_{q, r} \widetilde{F}_{k}^{h}, k=1,2, \forall h \in \mathbf{N}^{*} .
$$

Let us denote by $0_{k}^{h}$ the null matrix with $k$ rows and $h$ columns and by $E_{k}^{h}$ the $k \times h$ matrix

$$
E_{k}^{h}=\left\{\begin{array}{cc}
{\left[\begin{array}{cc}
I_{k} & 0_{k}^{h-k}
\end{array}\right]} & \text { if } h>k \\
I_{k} & \text { if } h=k \\
{\left[\begin{array}{c}
I_{k} \\
0_{k-h}^{h}
\end{array}\right]} & \text { if } h<k .
\end{array}\right.
$$

\section{Algorithm 5.4. (of minimal realization)}

Stage I. Expand $T(s, z)$ in Laurent series (4.9) and determine the Markov parameters $M_{i, j}$.

Stage II. Determine the degrees $q$ and $r$ of $p_{1}(s)$ and $p_{2}(z)$ where $p_{1}(s) p_{2}(z)$ is the l.c.d. of elements of $T(s, z)$.

Stage III. Write the two level block Hankel matrix $H_{q, r}$ and the matrix $K=\left[\begin{array}{cc}I_{\bar{p}} & H_{q, r} \\ 0 & I_{\bar{m}}\end{array}\right]$ where $\bar{p}=p q r$ and $\bar{m}=m q r$.

Stage IV. By applying elementary row operations on the first $\bar{p}$ rows of $K$ and elementary column operations on the last $\bar{m}$ columns of $K$, transform $K$ into

$$
\bar{K}=\left[\begin{array}{cc}
P & \bar{H} \\
0 & M
\end{array}\right] \quad \text { where } \quad \bar{H}=\left[\begin{array}{ll}
I_{n} & 0_{n}^{\bar{m}-n} \\
0_{\bar{p}-n}^{n} & 0_{\bar{p}-n}^{\bar{m}-n}
\end{array}\right] .
$$

Stage V. Calculate the minimal realization $\Sigma=\left(A_{1}, A_{2}, B, C\right)$ by the formula

$$
\begin{aligned}
& A_{k}=E_{n}^{\bar{p}} P\left[\sigma_{k} H_{q, r}\right] M E_{\bar{m}}^{n}, \quad k=1,2 ; \\
& B=E_{n}^{\bar{p}} P H_{q, r} E_{\bar{m}}^{m} ; C=E_{p}^{\bar{p}} H_{q, r} M E_{\bar{m}}^{n} .
\end{aligned}
$$

Proof. By elementary operations considerations it results that $P$ and $M$ are nonsingular since they are products of elementary matrices and that $P H_{q, r} M=\bar{H}=E_{\bar{p}}^{n} E_{n}^{\bar{m}}$. Moreover, the matrix $Q=M E_{\bar{m}}^{n} E_{n}^{\bar{p}} P$ is the pseudoinverse of $H_{q, r}$, i.e.

$$
H_{q, r} Q H_{q, r}=H_{q, r} .
$$

Firstly let us show that $A_{1}$ and $A_{2}$ are commutative matrices. We have by (5.4) and (5.8) $A_{1} A_{2}=\left(E_{n}^{\bar{p}} P\left[\sigma_{1} H_{q, r}\right] M E_{\bar{m}}^{n}\right)\left(E_{n}^{\bar{p}} P\left[\sigma_{2} H_{q, r}\right] M E_{\bar{m}}^{n}\right)=$ 
$E_{n}^{\bar{p}} P F_{1} H_{q, r} Q H_{q, r} \widetilde{F}_{2} M E_{\bar{m}}^{n}=E_{n}^{\bar{p}} P F_{1} H_{q, r} \widetilde{F}_{2} M E_{\bar{m}}^{n}=E_{n}^{\bar{p}} P F_{1} F_{2} H_{q, r} M E_{\bar{m}}^{n}$ and $A_{2} A_{1}$ has the same expression since $F_{1}$ and $F_{2}$ are commutative matrices.

In a similar way we can prove by induction that

$$
A_{1}^{i} A_{2}^{j}=E_{n}^{\bar{p}} P F_{1}^{i} F_{2}^{j} H_{q, r} M E_{\bar{m}}^{n} \quad \forall i, j \geq 0 .
$$

Now we can prove that $M_{i, j}=C A_{1}^{i} A_{2}^{j} B \quad \forall i, j \geq 0$. Indeed, by (5.7), (5.8), (5.9) and (5.5) we obtain $C B=\left(E_{p}^{\bar{p}} H_{q, r} M E_{\bar{m}}^{n}\right)\left(E_{n}^{\bar{p}} P H_{q, r} E_{\bar{m}}^{m}\right)=$ $E_{p}^{\bar{p}} H_{q, r} Q H_{q, r} E_{\bar{m}}^{m}=E_{p}^{\bar{p}} H_{q, r} E_{\bar{m}}^{m}=M_{0,0} ; C A_{1}^{i} A_{2}^{j} B=\left(E_{p}^{\bar{p}} H_{q, r} M E_{\bar{m}}^{n}\right)$ $\left(E_{n}^{\bar{p}} P F_{1}^{i} F_{2}^{j} H_{q, r} M E_{\bar{m}}^{n}\right)\left(E_{n}^{\bar{p}} P H_{q, r} E_{\bar{m}}^{m}\right)=\left(E_{p}^{\bar{p}} H_{q, r} M Q F_{1}^{i} F_{2}^{j} H_{q, r} Q H_{q, r} E_{\bar{m}}^{m}\right)=$ $\left(E_{p}^{\bar{p}} H_{q, r} M Q H_{q, r} \widetilde{F}_{1}^{i} \widetilde{F}_{2}^{j} E_{\bar{m}}^{m}\right)=\left(E_{p}^{\bar{p}} \sigma_{1}^{i} \sigma_{2}^{j} H_{q, r} E_{\bar{m}}^{m}\right)=M_{i, j} \forall i, j \geq 0$. Вy Proposition $4.4 \Sigma(5.7)$ is a realization of $T(s, z)$.

Since $P$ and $M$ are nonsingular matrices and $P H_{q, r} M=\bar{H}$ it results that $\operatorname{rank} H_{q, r}=\operatorname{rank} \bar{H}=n=\operatorname{dim} \Sigma$. By Proposition 5.1 we get $\operatorname{dim} \Sigma=\operatorname{rank} H_{q, r} \leq \operatorname{dim} \widetilde{\Sigma}$ for any realization $\Sigma$ of $T(s, z)$, hence the realization $\Sigma(5.7)$ is minimal.

\section{References}

[1] B. De Schutter, "Minimal state-space realization in linear system theory: an overview", Journal of Computational and Applied Mathematics, 121 (2000), 331-354.

[2] G.E. Antoniou, P.N. Paraskevopoulous, and S.J. Varoufakis, "Minimal statespace realization of factorable 2-D transfer functions", IEEE Trans. Circuits Systems, 35, 8 (1988), 1055-1058.

[3] S.H. Mentzelopoulou and N. J. Theodorou, "n-Dimensional Minimal State-Space Realization", IEEE Trans. Circuits Systems, 38, 3 (1991), 340-343.

[4] S.A. Miri and J.D. Aplevich, "Modeling and Realization of n-Dimensional Linear Discrete Systems", Multidimensional Systems and Signal Processing, 9 (1998), 241-253.

[5] B. L. Ho and R.E. Kalman, "Effective construction of linear, state-variable models from input/output functions", Regelungstechnik, 14, 12 (1966), 545-548.

[6] Z. Szabó, P.S.C. Heuberger, J. Bokor and P.M.J. Van den Hof, "Extended HoKalman algorithm for systems represented in generalized orthonormal bases", Automatica, 36 (2000), 1809-1818.

[7] V. Prepeliţă, "Systèmes linéaires à N indices". C.R. Acad. Sc. Paris, 279 (1974), 387-390.

[8] S. Attasi, "Introduction d'une classe de systèmes linéaires reccurents à deux indices". C.R. Acad. Sc. Paris, 277 (1973), 1135.

[9] V. Prepeliţă, "Linear hybrid systems", Bull. Math.Soc. Sci. Math. de Roumanie, 23 (71), 4 (1979), 391-403.

[10] R.P. Roesser, "A Discrete State-Space Model for Linear Image Processing", IEEE Trans. Aut. Control, AC-20 (1975), 1-10. 
[11] E. Fornasini and G. Marchesini, "State Space Realization Theory of TwoDimensional Filters", IEEE Trans.Aut.Control, AC-21 (1976), 484-492.

[12] T. Kaczorek, "Controllability and minimum energy control of 2D continuousdiscrete linear systems", Appl. Math. and Comp. Sci., 5, 1 (1995), 5-21.

[13] T. Kaczorek, "Singular Two-Dimensional Continuous-Discrete Linear Systems", Dynamics of Continuous, Discrete and Impulsive Systems, 2 (1996), 193-204.

[14] T. Kaczorek, Positive $1 D$ and $2 D$ Systems, Springer, London Berlin Heidelberg, 2002.

[15] K. Galkovski, E. Rogers and D.H. Owens, "New 2D models and a transition matrix for discrete linear repetitive processes", Int.J. Control, 72, 15 (1999), 1365-1380.

[16] J. Kurek and M.B. Zaremba, "Iterative learning control synthesis on 2D system theory", IEEE Trans.Aut.Control, AC-38, 1 (1993), 121-125.

[17] P. Picard, J. F. Lafay and V. Kucera, "Model Matching for Linear Systems with Delays and 2D Systems", Automatica, 34, 2 (1998), 183-191.

[18] V. Prepeliţă, "Generalized Dynamical systems", Scientific Bulletin, University Politehnica of Bucharest, 53, 3-4 (1991), 257-268.

[19] V. Prepeliţă and C. Drăguşin, "Linear Boundary Value Systems in the Space of Regulated Functions", Qualitative Problems for Differential Equations and Control Theory, C. Corduneanu (Ed.), World Scientific Publishing Co., (1995), 185-196.

[20] V. Prepeliţă, "Calculus of the fundamental matrix for generalized systems of differential equations", Annales Sci.Math. Québec, 23, 1 (1999), 87-96.

[21] V. Prepeliţă, "Criteria of reachability for 2D continuous-discrete systems", To appear in Rev. Roumaine Math. Pures Appl.

[22] V. Prepeliţă and Monica Pârvan, "Observability of 2D continuous-discrete separable systems", To appear in Rev. Roumaine Math. Pures Appl. 